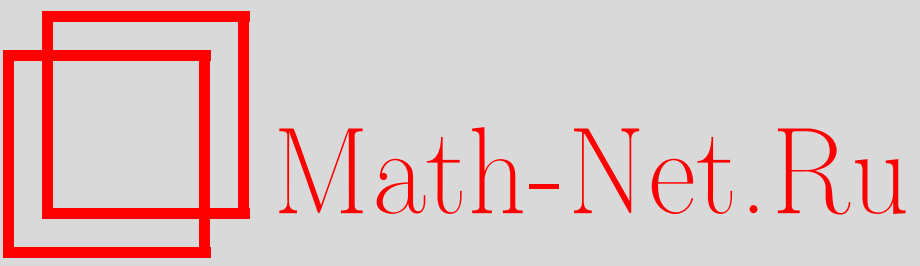

П. В. Тумаркин, Гиперболические $n$-мерные многогранники Кокстера с $n+3$ гипергранями, УМH, 2003, том 58, выпуск 4, 161-162

DOI: https://doi.org/10.4213/rm655

Использование Общероссийского математического портала Math-Net.Ru подразумевает, что вы прочитали и согласны с пользовательским соглашением

http://www.mathnet.ru/rus/agreement

Параметры загрузки:

IP : 34.239 .49 .27

26 апреля 2023 г., 18:18:49 


\section{ГИПЕРБОЛИЧЕСКИЕ $n$-МЕРНЫЕ МНОГОГРАННИКИ КОКСТЕРА С $n+3$ ГИПЕРГРАНЯМИ}

\section{П. В. ТУМАРКИН}

1. Выпуклый многогранник называется многогранником Кокстера, если все его двугранные углы являются целшми частями $\pi$. Группа, порожденная отражениями относительно гиперграней произвольного многогранника Кокстера, дискретна, причем фундаментальной областью такой группы является сам многогранник. Многогранники Кокстера удобно описьвать схемами Кокстера [1].

Наиболшший интерес представляют гиперболические многогранники Кокстера конечного объема. В отличие от сферического и параболического случаев, полной классификации таких многогранников нет. Известно, что размерность ограниченного многогранника Кокстера не превышает 29 [2], а неограниченного - 995 [3]. Полностью охарактеризованы многогранники Кокстерав $\mathbb{H}^{3}[4],[5] ;$ перечислены также всегиперболические симплексы [6], [7] и многогранники с $n+2$ гипергранями [8]-[10].

В [9] доказано, что размерность ограниченного $n$-мерного гиперболического многогранника Кокстера с $n+3$ гипергранями не превышает 8 . Известен пример неограниченного многогранника в $\mathbb{H}^{15}$ с 18 гипергранями (см. [1]). Основным результатом данной работы является

ТеОрема 1. В гиперболическом пространстве размерности $n \geqslant 17$ не существует многогранников Кокстера конечного объема с $n+3$ гранями. $B \mathbb{H}^{16}$ существует ровно один такой многогранник; он имеет следующую схему Кокстера:

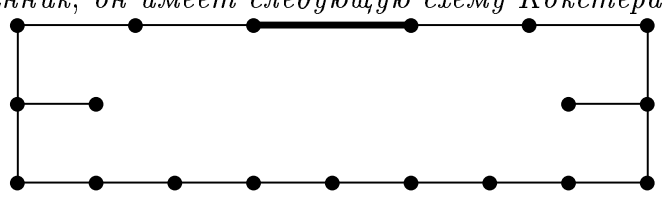

2. Каждому комбинаторному типу $n$-мерного многогранника с $n+3$ гипергранями соответствует стандартная двумерная диаграмма Гейла (см., например, [11]). Стандартная диаграмма Гейла $n$-мерного многогранника с $n+3$ гранями, не являющегося пирамидой, представляет собой правильньй $2 k$-угольник со взвешенными вершинами с центром в начале координат. Веса удовлетворяют следующим условиям:

1) веса являются целыми неотрицательными числами, и их сумма равна $n+3$, причем веса никаких двух соседних (а также противоположных) вершин не равны нулю одновременно;

2) для любой прямой, проходящей через начало координат, сумма весов вершин, лежащих в каждой открытой полуплоскости, ограниченной этой прямой, не меньше двух.

Комбинаторньй тип многогранника $P$ можно восстановить по диаграмме Гейла следующим образом. Вершине многоугольика $a_{i}$ с весом $\mu_{i}$ соответствует $\mu_{i}$ гиперграней $P f_{i, 1}, \ldots, f_{i, \mu_{i}}$. Для любого подмножества $I$ множества гиперграней многогранника $P$ пересечение набора гиперграней $\left\{f_{j, \gamma} \mid(j, \gamma) \in I\right\}$ является гранью $P$ тогда и только тогда, когда начало координат лежит в выпуклой оболочке множества $\widehat{I}=\left\{a_{j} \mid(j, \gamma) \notin I\right\}$. Начало координат лежит в относительной внутренности вьпуклой оболочки множества $\widehat{I}$ тогда и только тогда, когда $I$ - максимальный (по включению) набор гиперграней, дающий в пересечении данную грань.

Ланнеровской (квази-ланнеровской) схемой назьвается связная схема Кокстера, которая не является ни эллиптической, ни параболической, но любая ее собственная подсхема - эллиптическая (эллиптическая или параболическая).

Если $G$ - диаграмма Гейла многогранника $P$, то через $S_{m, l}$ мы будем обозначать подсхему схемы Кокстера $S(P)$, соответствующую последовательньм $l-m+1(\bmod 2 k)$ вершинам $a_{m}, \ldots, a_{l}$ диаграммы $G$.

Следующие две леммы выводятся непосредственно из определения диаграммы Гейла и теорем 3.1 и 3.2 работы [1]. 
Лемма 1. Пусть $G$ - диаграмма Гейла многогранника $P$. Пусть веса точек $a_{i}, a_{k+i}$ отличны от нуля. Тогда

1) веса точек $a_{i}$ и $a_{k+i}$ равны 1 , а схемы Кокстера $S_{i+1, k+i-1} u S_{k+i+1, i-1}$ являются связными параболическими;

2) если веса точек $a_{i+1}$ и $a_{k+i+1}$ не равны нулю, то схема $S_{i+1, k+i}$ является квази-ланнеровской;

3) если вес точки $a_{i+1}$ равен нулю, то схема Кокстера $S_{i+2, k+i}$ является квази-ланнеровской.

Лемма 2. Пусть $G$ - диаграмма Гейла многогранника $P$. Пусть веса точек $a_{i} u$ $a_{k+i-1}$ равны нулю. Тогда схема Кокстера $S_{i+1, k+i-2}$ является ланнеровской.

Поскольку число вершин в ланнеровской диаграмме не превосходит 5 [6], а в квази-ланнеровской - 10 [7], из леммы 2 и утверждений 2) и 3 ) леммы 1 следует

Лемма 3. Пусть $G$ - диаграмма Гейла многогранника $P$ и веса точек $a_{i}$ и $a_{i+2}$ равны нулю. Тогда сумма весов всех вершин не превышает 20.

Неболшшая проверка оставшихся случаев показьвает, что размерность многогранника не может превышать 17. Поэтому число вершин (с кратностями) с ненулевыми весами в диаграмме Гейла не превосходит 20 . С другой стороны, при достаточно большом $k$ и достаточно болшшом количестве вершин с нулевыми весами соответствующая схема Кокстера содержит не менее двух ланнеровских подсхем. В последнем случае $n \leqslant 15$. Отсюда следует ограничение на $k$ : если $n \geqslant 16$, то $k \leqslant 13$. Перебор оставшихся случаев показьвает, что при $n=17$ исследуемшхх многогранников нет, а при $n=16$ такой многогранник ровно один.

Осталось разобрать случай, когда $P$ является пирамидой. Используя тот факт, что двукратная пирамида в пространстве Лобачевского не может быть многогранником Кокстера [10], несложно доказать следующую лемму.

Лемма 4. Если п-мерный гиперболический многогранник Кокстера конечного оббема с $n+3$ гранями является пирамидой, то это пирамида над произведением трех симплексов.

Все такие многогранники несложно перечислить, их размерность не превьшает 11.

Автор благодарит Э.Б. Винберга за внимание к работе и полезные замечания.

\section{СПИСОК ЛИТЕРАТУРЫ}

[1] Э. Б. Винберг // УМН. 1985. Т. 40. №1. С. 29-66. [2] Э. Б. Винберг // Труды ММО. 1984. Т. 47. С. 68-102. [3] М. Н. Прохоров // Изв. АН СССР. Сер. матем. 1986. Т. 50. № 2. С. 413-424. [4] Е. М. Андреев // Матем. сб. 1970. Т. 81. №3. С. 445-478. [5] Е. М. Андреев // Матем. сб. 1970. T. 83. № 2. C. 256-260. [6] F. Lannér // Comm. Sem. Math. Univ. Lund. 1950. V. 11. Р. 1-71. [7] Э. Б. Винберг // Матем. сб. 1967. Т. 72. №3. С. 471-488; Т. 73. № 2. С. 303. [8] И. М. Каплинская // Матем. заметки. 1974. Т. 15. №1. С. 159-164. [9] F. Esselmann. Über kompakte hyperbolische Coxeter-Polytope mit wenigen Facetten // Preprint No. 94-087. Bielefeld: Universität Bielefeld, SFB 343. [10] P. Tumarkin. Hyperbolic Coxeter $n$-polytopes with $n+2$ facets // math.MG/0301133. [11] B. Grünbaum. Convex Polytopes. London: Wiley, 1967.

Московский государственньй

Принято редколлегией университет им. М. В. Ломоносова 14.05 .2003 